\title{
Restricted extension of sparse partial edge colorings of complete graphs
}

\author{
Carl Johan Casselgren* \\ Department of Mathematics \\ Linköping University \\ SE-581 83 Linköping, Sweden \\ carl.johan. casselgren@liu.se
}

\author{
Lan Anh Pham \\ Department of Mathematics \\ Umeå University \\ SE-901 87 Umeå, Sweden \\ lan.pham@umu.se
}

Submitted: Apr 29, 2020; Accepted: Feb 26, 2021; Published: Apr 9, 2021

(C) The authors. Released under the CC BY-ND license (International 4.0).

\begin{abstract}
Given a partial edge coloring of a complete graph $K_{n}$ and lists of allowed colors for the non-colored edges of $K_{n}$, can we extend the partial edge coloring to a proper edge coloring of $K_{n}$ using only colors from the lists? We prove that this question has a positive answer in the case when both the partial edge coloring and the color lists satisfy certain sparsity conditions.
\end{abstract}

Mathematics Subject Classifications: 05C15, 05B15

\section{Introduction}

An edge precoloring (or partial edge coloring) of a graph $G$ is a proper edge coloring of some subset $E^{\prime} \subseteq E(G)$; a $t$-edge precoloring is such a coloring with $t$ colors. A $t$-edge precoloring $\varphi$ is extendable if there is a proper $t$-edge coloring $f$ such that $f(e)=\varphi(e)$ for any edge $e$ that is colored under $\varphi ; f$ is called an extension of $\varphi$. In general, the problem of extending a given edge precoloring is an $\mathcal{N} \mathcal{P}$-complete problem, already for 3-regular bipartite graphs, as proved by Fiala [13].

Questions on extending a partial edge coloring seem to have been first considered for balanced complete bipartite graphs, and these questions are usually referred to as problems on completing partial Latin squares. In this form the problem appeared already in 1960, when Evans [12] stated his now classic conjecture that for every positive integer $n$, if $n-1$ edges in $K_{n, n}$ have been (properly) colored, then the partial coloring can be extended to a proper $n$-edge-coloring of $K_{n, n}$. This conjecture was solved for large $n$ by

\footnotetext{
${ }^{*}$ Casselgren was supported by a grant from the Swedish Research Council (2017-05077).
} 
Häggkvist [15] and later for all $n$ by Smetaniuk [18], and independently by Andersen and Hilton [1]. Similar questions have also been investigated for complete graphs by Andersen and Hilton [2]; as is well-known, problems on extending partial edge colorings of complete graphs can be formulated as questions on completing symmetric partial Latin squares. Moreover, quite recently, Casselgren et al. [8] proved an analogue of this result for hypercubes.

Generalizing this problem, Daykin and Häggkvist [11] proved several results on extending partial edge colorings of $K_{n, n}$, and they also conjectured that much denser partial colorings can be extended, as long as the colored edges are spread out in a specific sense: a partial $n$-edge coloring of a graph is $\epsilon$-dense if there are at most $\epsilon n$ colored edges from $\{1, \ldots, n\}$ at any vertex and each color in $\{1, \ldots, n\}$ is used at most $\epsilon n$ times in the partial coloring. Daykin and Häggkvist [11] conjectured that for every positive integer $n$, every $\frac{1}{4}$-dense partial proper $n$-edge coloring of $K_{n, n}$ can be extended to a proper $n$-edge coloring of $K_{n, n}$, and proved a version of the conjecture for $\epsilon=o(1)$ (as $n \rightarrow \infty$ ) and $n$ divisible by 16. Bartlett [7] proved that this conjecture holds for a fixed positive $\epsilon$, and recently a different proof which improves the value of $\epsilon$ was given by Barber et al [6].

For general edge colorings of balanced complete bipartite graphs, Dinitz conjectured, and Galvin [14] proved, that if each edge of $K_{n, n}$ is given a list of $n$ colors, then there is a proper edge coloring of $K_{n, n}$ with support in the lists. Indeed, Galvin's result was a complete solution of the well-known List Coloring Conjecture for the case of bipartite multigraphs (see e.g. [10] for more background on this conjecture and its relation to the Dinitz conjecture).

Motivated by the Dinitz problem, Häggkvist [16] introduced the notion of $\beta n$-arrays, which correspond to list assignments $L$ of forbidden colors for $E\left(K_{n, n}\right)$, such that each edge $e$ of $K_{n, n}$ is assigned a list $L(e)$ of at most $\beta n$ forbidden colors from $\{1, \ldots, n\}$, and at every vertex $v$ each color is forbidden on at most $\beta n$ edges incident to $v$; we call such a list assignment (of any graph), with colors from some base set $\{1, \ldots, n\}, \beta$-sparse. If $L$ is a list assignment for $E\left(K_{n, n}\right)$, then a proper $n$-edge coloring $\varphi$ of $K_{n, n}$ avoids the list assignment $L$ if $\varphi(e) \notin L(e)$ for every edge $e$ of $K_{n, n}$; if such a coloring exists, then $L$ is avoidable. Häggkvist conjectured that there exists a fixed $\beta>0$, in fact also that $\beta=\frac{1}{3}$, such that for every positive integer $n$, every $\beta$-sparse list assignment for $K_{n, n}$ is avoidable. That such a $\beta>0$ exists was proved for even $n$ by Andrén in her $\mathrm{PhD}$ thesis [3], and later for all $n$ by Andrén et al [4].

Combining the notions of extending a sparse precoloring and avoiding a sparse list assignment, Andrén et al. [5] proved that there are constants $\alpha>0$ and $\beta>0$, such that for every positive integer $n$, every $\alpha$-dense partial edge coloring of $K_{n, n}$ can be extended to a proper $n$-edge-coloring avoiding any given $\beta$-sparse list assignment $L$, provided that no edge $e$ is precolored by a color that appears in $L(e)$. Quite recently, Casselgren et al [9] obtained analogous results for hypercubes. Moreover, similar results for a more general family of graphs have been proved by Pham [17].

In this paper, we consider the corresponding problem for complete graphs. As mentioned above, edge precoloring extension problems have previously been considered for complete graphs; the type of questions that we are interested in here, however, seems to 
be a hitherto quite unexplored line of research.

For an integer $p$, we define $t=4 r-1$ if $p=4 r$ or $p=4 r-1$, and $t=4 r-2$ if $p=4 r-2$ or $p=4 r-3$. Our main result is the following.

Theorem 1. There are constants $\alpha>0$ and $\beta>0$ such that for every positive integer $p$, if $\varphi$ is an $\alpha$-dense $t$-edge precoloring of $K_{p}, L$ is a $\beta$-sparse list assignment from the color set $\{1, \ldots, t\}$, and $\varphi(e) \notin L(e)$ for every edge $e \in E\left(K_{p}\right)$, then there is a proper $t$-edge coloring of $K_{p}$ which agrees with $\varphi$ on any precolored edge and which avoids $L$.

The number of colors in Theorem 1 agrees with the chromatic index of the complete graph if $p \in\{4 r, 4 r-1\}$ and is thus best possible; we do not know whether $t=4 r-2$ can be replaced by $t=4 r-3$ if $p \in\{4 r-2,4 r-3\}$. In fact, the number of colors used in Theorem 1 is due to the proof method used in this paper: the general proof method in the papers $[9,5,4,7]$ rely on the existence of a proper edge coloring of the considered graph where every (or almost every) edge is contained in a large number of 2-colored 4-cycles. Roughly speaking, after applying a simple probabilistic argument, the idea is then to switch colors on such 4-cycles in a systematic way, so that the resulting coloring agrees with the precoloring and respects the colors forbidden by the list assignment. Applying similar methods to complete graphs requires a proper edge coloring where all or ("almost all") edges are contained in a large number of 2-edge-colored 4-cycles. However, we do not know of any such proper edge coloring of a complete graph; thus, to be able to apply methods previously used for complete bipartite graphs, we decompose a complete graph $K_{2 n}$ of order $2 n$ into two copies of $K_{n}$ and a complete bipartite graph $K_{n, n}$. In particular, this means that a large number of edges in $K_{2 n}$ are not contained in 2-edgecolored 4-cycles, but every edge is adjacent to "many" edges that are contained in such 4-cycles. Nevertheless, to be able to apply the machinery from [5], we need to significantly strengthen these techniques.

Since any complete graph $K_{2 n-1}$ of odd order is a subgraph of $K_{2 n}$, the following theorem implies Theorem 1.

If $n$ is even, let $m=2 n-1$, and if $n$ is odd, let $m=2 n$.

Theorem 2. There are constants $\alpha>0$ and $\beta>0$ such that for every positive integer $n$, if $\varphi$ is an $\alpha$-dense $m$-edge precoloring of $K_{2 n}, L$ is a $\beta$-sparse list assignment for $K_{2 n}$ from the color set $\{1, \ldots, m\}$, and $\varphi(e) \notin L(e)$ for every edge $e \in E\left(K_{2 n}\right)$, then there is a proper $m$-edge coloring of $K_{2 n}$ which agrees with $\varphi$ on any precolored edge and which avoids $L$.

The rest of the paper is devoted to the proof of Theorem 2. As already mentioned, the proof of this theorem uses the same strategy as the proof of the main result of [5], and we shall need to adapt several tools from $[5,7]$ to the setting of complete graphs.

\section{Terminology, notation and proof outline}

Let $\left\{p_{1}, p_{2}, \ldots, p_{n}, q_{1}, q_{2}, \ldots, q_{n}\right\}$ be the $2 n$ vertices of the complete graph $K_{2 n}$, and let $G_{1}$ be the subgraph induced by $\left\{p_{1}, p_{2}, \ldots, p_{n}\right\}$, and $G_{2}$ be the subgraph induced by 
$\left\{q_{1}, q_{2}, \ldots, q_{n}\right\} ;$ so $G_{1}$ and $G_{2}$ are both isomorphic to $K_{n}$. We denote by $K_{n, n}$ the graph $K_{2 n}-E\left(G_{1}\right) \cup E\left(G_{2}\right)$, so $K_{n, n}$ is the complete bipartite graph with partite sets $\left\{p_{1}, p_{2}, \ldots, p_{n}\right\}$ and $\left\{q_{1}, q_{2}, \ldots, q_{n}\right\}$. For any proper edge coloring $h$ of $K_{2 n}$, we denote by $h_{K}$ the restriction of this coloring to $K_{n, n}$; similarly, $h_{G_{1}}$ and $h_{G_{2}}$ are the restrictions of $h$ to the subgraphs $G_{1}$ and $G_{2}$, respectively.

For a vertex $u \in V\left(K_{2 n}\right)$, we denote by $E_{u}$ the set of edges with one endpoint being $u$, and for a (partial) edge coloring $f$ of $K_{2 n}$, let $f(u)$ denote the set of colors on the edges in $E_{u}$ under $f$. Let $\varphi$ be an $\alpha$-dense precoloring of $K_{2 n}$. Edges of $K_{2 n}$ which are colored under $\varphi$, are called prescribed (with respect to $\varphi$ ). For an edge coloring $h$ of $K_{2 n}$, an edge $e$ of $K_{2 n}$ is called requested (under $h$ with respect to $\varphi$ ) if $h(e)=c$ and $e$ is adjacent to an edge $e^{\prime}$ such that $\varphi\left(e^{\prime}\right)=c$.

Consider a $\beta$-sparse list assignment $L$ for $K_{2 n}$. For an edge coloring $h$ of $K_{2 n}$, an edge $e$ of $K_{2 n}$ is called a conflict edge (of $h$ with respect to $L$ ) if $h(e) \in L(e)$; such edges are also referred to as just conflicts. An allowed cycle (under $h$ with respect to $L$ ) of $K_{2 n}$ is a 4-cycle $\mathcal{C}=$ uvztu in $K_{2 n}$ that is 2-colored under $h$, and such that interchanging colors on $\mathcal{C}$ yields a proper edge coloring $h_{1}$ of $K_{2 n}$ where none of $u v, v z, z t, t u$ is a conflict edge. We call such an interchange a swap on $h$, or a swap on $\mathcal{C}$.

Let us now outline the proof of Theorem 2.

Step I. Define a standard $m$-edge coloring $h$ of the complete graph $K_{2 n}$. In particular, this coloring has the property that "most" edges of $K_{n, n}$ are contained in a large number of 2-colored 4-cycles.

Step II. Given the standard $m$-edge coloring $h$ of $K_{2 n}$, from $h$ we construct a new proper $m$-edge-coloring $h^{\prime}$ that satisfies certain sparsity conditions; in particular every vertex of $K_{2 n}$ is incident with a "small" number of conflict edges, and every color class of $h^{\prime}$ contains a "small" number of conflict edges. These sparsity conditions will enable use to apply a modified variant of the machinery from $[5,7]$ for finding a coloring that agrees with $\varphi$ and which avoids $L$.

The exact formulation of these conditions shall be given below.

Step III. From the precoloring $\varphi$ of $K_{2 n}$, we define a new edge precoloring $\varphi^{\prime}$ that agrees with $\varphi$, and such that an edge $e$ of $K_{2 n}$ is colored under $\varphi^{\prime}$ if and only if $e$ is colored under $\varphi$ or $e$ is a conflict edge of $h^{\prime}$ with respect to $L$. As for $\varphi$, we shall also require that each of the colors in $\{1, \ldots, m\}$ is used a bounded number of times under $\varphi^{\prime}$.

Step IV. In this step we prove a series of lemmas which roughly implies that for almost all pairs of edges $e$ and $e^{\prime}$ in $K_{2 n}$, we can construct a new edge coloring $h^{T}$ from $h^{\prime}$ (or a coloring obtained from $h^{\prime}$ ) such that $h^{T}\left(e^{\prime}\right)=h^{\prime}(e)$ by recoloring a "small" subgraph of $K_{2 n}$. This property is crucial for our recoloring procedure for obtaining a proper edge coloring of $K_{2 n}$ that agrees with $\varphi$ and which avoids $L$, which is described in the next step. 
Step V. Using the lemmas proved in the previous step, we shall in this step from $h^{\prime}$ construct a coloring $h_{q}$ of $K_{2 n}$ that agrees with $\varphi^{\prime}$ and which avoids $L$. This is done iteratively by steps: in each step we consider a prescribed edge $e$ of $K_{2 n}$, such that $h^{\prime}(e) \neq \varphi^{\prime}(e)$, and construct a subgraph $T_{e}$ of $K_{2 n}$, such that performing a series of swaps on allowed cycles, all edges of which are in $T_{e}$, we obtain a coloring $h_{e}$ where $h_{e}(e)=\varphi^{\prime}(e)$. Hence, after completing this iterative procedure we obtain a coloring that is an extension of $\varphi^{\prime}$ (and thus $\varphi$ ), and which avoids $L$.

The main idea of our proof is thus to use structural properties of the restriction of the coloring $h$ to $K_{n, n}$ for making stepwise alterations of the coloring $h$ of $K_{2 n}$. Since the restriction of $h$ to $G_{1}\left(G_{2}\right)$ does not satisfy any such strong structural properties, we need to extend the general method from [5]. Thus, the major differences between our proof and the proof of the main result in [5] are in Steps IV and V, and the proofs in these steps require a significant generalization of the machinery used in $[5,7]$ to the setting of complete graphs. On the other hand, the proofs in Steps I-III are very similar (or even identical) to the proofs in [5]; thus, we shall in general omit the proofs in these steps.

\section{Proofs}

In this section we prove Theorem 2. In the proof we shall verify that it is possible to perform Steps I-V described above to obtain a proper $m$-edge-coloring of $K_{2 n}$ that is an extension of $\varphi$ and which avoids $L$. This is done by proving some lemmas in each step.

The proof of Theorem 2 involves a number of functions and parameters:

$$
\alpha, \beta, d, \epsilon, k, c(n), f(n)
$$

and a number of inequalities that they must satisfy. For the reader's convenience, explicit choices for which the proof holds are presented here:

$$
\begin{gathered}
\alpha=\frac{1}{1000000}, \quad \beta=\frac{1}{1000000}, \quad d=\frac{1}{200}, \quad \epsilon=\frac{1}{50000}, \\
k=\frac{1}{5000}, \quad c(n)=\left\lfloor\frac{n}{50000}\right\rfloor, \quad f(n)=\left\lfloor\frac{n}{10000}\right\rfloor .
\end{gathered}
$$

We shall also use the functions

$$
c^{\prime}(n)=c(n) / 2, \quad H(n)=9 \alpha m+9 f(n)+6 c(n)+4 d n, \quad P(n)=d n+\alpha m+f(n) .
$$

Furthermore, we shall assume that $n$ is large enough whenever necessary. Since the proof contains a finite number of inequalities that are valid if $n$ is large enough, say $n \geqslant N$, this suffices for proving the theorem with $\alpha^{\prime}$ and $\beta^{\prime}$ in place of $\alpha$ and $\beta$, and where we set $\alpha^{\prime}=\min \{1 / N, \alpha\}$ and $\beta^{\prime}=\min \{1 / N, \beta\}$.

We remark that since the numerical values of $\alpha$ and $\beta$ are not anywhere near what we expect to be optimal, we have not put an effort into choosing optimal values for these 
parameters; see [9] for a more elaborate discussion on upper bounds for $\alpha$ and $\beta$ that hold for any $d$-regular graph.

Finally, for simplicity of notation, we shall omit floor and celling signs whenever these are not crucial.

Proof of Theorem 2. Let $\varphi$ be an $\alpha$-dense precoloring of $K_{2 n}$, and let $L$ be a $\beta$-sparse list assignment for $K_{2 n}$ such that $\varphi(e) \notin L(e)$ for every edge $e \in E\left(K_{2 n}\right)$.

Step I: Below we shall define the standard $m$-edge coloring $h$ of the complete graph $K_{2 n}$ by defining an $n$-edge coloring for $K_{n, n}$ using the set of colors $\{1,2, \ldots, n\}$ and a $(m-n)$ edge coloring for $G_{1}$ and $G_{2}$ using the set of colors $\{n+1, \ldots, m\}$. Throughout this paper, we assume $x \bmod k=k$ in the case when $x \equiv 0 \bmod k$.

Firstly, we define a proper $n$-edge coloring for $K_{n, n}$ using the set of colors $\{1,2, \ldots, n\}$. This coloring was used in $[4,5,7]$, and we shall give the explicit construction for the case when $n$ is even. For the case $n$ is odd, one can modify the construction in the even case by swapping on some 2-colored 4-cycles and using a transversal; the details are given in Lemma 2.1 in [7].

So suppose that $n=2 r$. For $1 \leqslant i, j \leqslant n$, the standard coloring $h_{K}$ for $K_{n, n}$ is defined as follows.

$$
h_{K}\left(p_{i} q_{j}\right)=\left\{\begin{array}{lll}
j-i+1 & \bmod r & \text { for } i, j \leqslant r \\
i-j+1 & \bmod r & \text { for } i, j>r \\
(j-i+1 & \bmod r)+r & \text { for } i \leqslant r, j>r \\
(i-j+1 & \bmod r)+r & \text { for } i>r, j \leqslant r .
\end{array}\right.
$$

If a 2-colored 4-cycle with colors $c_{1}$ and $c_{2}$ satisfies that

$$
\left|\left\{c_{1}, c_{2}\right\} \cap\{1, \ldots, r\}\right|=1
$$

then $C$ is called a strong 2-colored 4-cycle. The following property of $h_{K}$ is fundamental for our proof.

Lemma 3. $[4,5,7]$ Each edge in $K_{n, n}$ belongs to exactly $r$ distinct strong 2-colored 4-cycles under $h_{K}$.

For the case when $n=2 r+1$, we can construct an $n$-edge coloring $h_{K}$ for $K_{n, n}$ such that all but at most $3 n+7$ edges are in $\left\lfloor\frac{n}{2}\right\rfloor$ strong 2 -colored 4 -cycles. In particular, there is a vertex in $K_{n, n}$ where no edge belongs to at least $\left\lfloor\frac{n}{2}\right\rfloor$ strong 2-colored 4-cycles. The full proof appears in [7] and therefore we omit the details here.

Secondly, let us define $(m-n)$-edge colorings of $G_{1}$ and $G_{2}$ using the set of colors $\{n+1, \ldots, m\}$. Suppose first that $n$ is odd, and recall that $m=2 n$. We define the colorings $h_{G_{1}}$ of $G_{1}$ and $h_{G_{2}}$ of $G_{2}$ by, for $1 \leqslant i, j \leqslant n$, setting

$$
h_{G_{1}}\left(p_{i} p_{j}\right)=h_{G_{2}}\left(q_{i} q_{j}\right)=(i+j \bmod n)+n .
$$

Assume now that $n$ is even, and recall that $m=2 n-1$. We define the colorings $h_{G_{1}}$ of $G_{1}$ and $h_{G_{2}}$ of $G_{2}$ as follows: 
- $h_{G_{1}}\left(p_{i} p_{j}\right)=h_{G_{2}}\left(q_{i} q_{j}\right)=(i+j \bmod n-1)+n$ for $1 \leqslant i, j \leqslant n-1$.

- $h_{G_{1}}\left(p_{i} p_{n}\right)=h_{G_{2}}\left(q_{i} q_{n}\right)=(2 i \bmod n-1)+n$ for $1 \leqslant i \leqslant n-1$.

It is straightforward to verify that $h_{K}, h_{G_{1}}, h_{G_{2}}$ are proper colorings. Taken together, the colorings $h_{K}, h_{G_{1}}, h_{G_{2}}$ constitute the standard $m$-edge coloring $h$ of $K_{2 n}$.

Step II: Let $h$ be the $m$-edge coloring of $K_{2 n}$ obtained in Step I, and let $\rho=\left(\rho_{1}, \rho_{2}\right)$ be a pair of permutations chosen independently and uniformly at random from all $n$ ! permutations of the vertex labels of $G_{1}$ and $n$ ! permutations of the vertex labels of $G_{2}$. We permute the labels of the vertices with respect to the coloring of $h$, while $\varphi$ is considered as a fixed partial coloring of $K_{2 n}$, as is also the list assignment $L$. Thus we can view a relabeling of the vertices in $G_{1}$ and $G_{2}$ with respect to $h$ (while keeping colors of edges fixed) as equivalent to defining a new proper edge coloring of $K_{2 n}$ from $h$ by recoloring edges in $K_{2 n}$. Hence, we can think of $\rho$ as being applied to the edge coloring $h$ of $K_{2 n}$ thereby defining a new edge coloring of $K_{2 n}$ (rather than permuting vertex labels).

Denote by $h^{\prime}$ a random $m$-edge coloring obtained from $h$ by applying $\rho$ to $h$. Note that if $u^{\prime}=\rho(u)$ and $v^{\prime}=\rho(v)$, then $h^{\prime}\left(u^{\prime} v^{\prime}\right)=h(u v)$.

Lemma 4. Suppose that $\alpha, \beta, \epsilon$ are constants, and $c(n)$ and $c^{\prime}(n)=c(n) / 2$ are functions of $n$, such that $n-1>2 c(n)>4$ and

$$
\begin{gathered}
\left(\frac{4 \beta}{\epsilon-4 \beta}\right)^{\epsilon-4 \beta}\left(\frac{1}{1-2 \epsilon+8 \beta}\right)^{1 / 2-\epsilon+4 \beta}<1, \\
\alpha, \beta<\frac{c(n)}{2(n-c(n))}\left(\frac{n-c(n)}{n}\right)^{\frac{n}{c(n)}}, \text { and } \\
\beta<\frac{c^{\prime}(n)}{2\left(n-c^{\prime}(n)\right)}\left(\frac{n-c^{\prime}(n)}{n}\right)^{\frac{n}{c^{\prime}(n)}} .
\end{gathered}
$$

Then the probability that $h^{\prime}$ fails the following conditions tends to 0 as $n \rightarrow \infty$.

(a) All edges in $K_{n, n}$, except for $3 n+7$, belong to at least $\left\lfloor\frac{n}{2}\right\rfloor-\epsilon n$ allowed strong 2-colored 4-cycles.

(b) Each vertex of $K_{n, n}$ is incident to at most $c^{\prime}(n)$ conflict edges in $K_{n, n}$.

(c) For each color $c \in\{1,2, \ldots, n\}$, there are at most $c(n)$ edges in $K_{n, n}$ that are colored $c$ that are conflicts.

(d) For each color $c \in\{1,2, \ldots, n\}$, there are at most $c(n)$ edges in $K_{n, n}$ that are colored $c$ that are prescribed.

(e) For each pair of colors $c_{1} \in\{1,2, \ldots, m\}$ and $c_{2} \in\{1,2, \ldots, n\}$, there are at most $c(n)$ edges $e$ in $K_{n, n}$ with color $c_{2}$ such that $c_{1} \in L(e)$.

(f) Each vertex of $G_{1}\left(G_{2}\right)$ is incident to at most $c^{\prime}(n)$ conflict edges in $G_{1}\left(G_{2}\right)$. 
(g) For each color $c \in\{n+1, n+2, \ldots, m\}$, there are at most $c(n)$ edges in $G_{1}\left(G_{2}\right)$ that are colored $c$ that are conflicts.

(h) For each color $c \in\{n+1, n+2, \ldots, m\}$, there are at most $c(n)$ edges in $G_{1}\left(G_{2}\right)$ that are colored $c$ that are prescribed.

(i) For each pair of colors $c_{1} \in\{1,2, \ldots, m\}$ and $c_{2} \in\{n+1, n+2, \ldots, m\}$, there are at most $c(n)$ edges e in $G_{1}\left(G_{2}\right)$ with color $c_{2}$ such that $c_{1} \in L(e)$.

The proof of this lemma is very similar to corresponding auxiliary results in [5]. By applying Lemmas $3.2,3.3,3.4$ in [5], we can immediately deduce that the probability that $h^{\prime}$ fails conditions (a), (b), (c), (d) or (e) tends to 0 as $n \rightarrow \infty$ if

$$
\begin{gathered}
\left(\frac{2 \beta^{\prime}}{\epsilon-2 \beta^{\prime}}\right)^{\epsilon-2 \beta^{\prime}}\left(\frac{1}{1-2 \epsilon+4 \beta^{\prime}}\right)^{1 / 2-\epsilon+2 \beta^{\prime}}<1 \\
\alpha^{\prime}, \beta^{\prime}<\frac{c(n)}{(n-c(n))}\left(\frac{n-c(n)}{n}\right)^{\frac{n}{c(n)}} ; \beta^{\prime}<\frac{c^{\prime}(n)}{\left(n-c^{\prime}(n)\right)}\left(\frac{n-c^{\prime}(n)}{n}\right)^{\frac{n}{c^{\prime}(n)}} .
\end{gathered}
$$

Since all these inequalities are true, it remains to prove that the probability that $h^{\prime}$ fails conditions (f), (g), (h) or (i) tends to 0 as $n \rightarrow \infty$.

However, that this indeed holds can be proved using arguments that are completely analogous to the proofs of Lemmas 3.3-3.4 in [5]. Hence, we omit the details.

Lemma 4 implies that there exists a pair of permutations $\rho=\left(\rho_{1}, \rho_{2}\right)$ such that if $h^{\prime}$ is the proper $m$-edge coloring obtained from $h$ by applying $\rho$ to $h$ then $h^{\prime}$ satisfies conditions (a)-(i) of Lemma 4; then the coloring $h^{\prime}$ also satisfies the following.

(a') Each vertex of $K_{2 n}$ is incident to at most $c(n)$ conflict edges;

(b') For each color $c \in\{1,2, \ldots, m\}$, there are at most $c(n)$ edges in $K_{2 n}$ that are colored $c$ that are conflicts (prescribed);

(c') For each pair of colors $c_{1}, c_{2} \in\{1,2, \ldots, m\}$, there are at most $c(n)$ edges $e$ in $K_{2 n}$ with color $c_{2}$ such that $c_{1} \in L(e)$.

Moreover, if we define $\alpha^{\prime}=2 \alpha$ and $\beta^{\prime}=2 \beta$; then the $\alpha$-dense precoloring $\varphi$ satisfies that

(I) every color appears on at most $\alpha^{\prime} n$ edges;

(II) for every vertex $v$, at most $\alpha^{\prime} n$ edges incident with $v$ are precolored.

Furthermore, for the $\beta$-sparse list assignment $L$, we have

(III) $|L(e)| \leqslant \beta^{\prime} n$ for every edge of $K_{2 n}$;

(IV) for every vertex $v$, every color appears in the lists of at most $\beta^{\prime} n$ edges incident to $v$. 
Step III: Let $h^{\prime}$ be the proper $m$-edge coloring satisfying conditions (a)-(i) of Lemma 4 obtained in the previous step.

We use the following lemma for extending $\varphi$ to a proper $m$-edge precoloring $\varphi^{\prime}$ of $K_{2 n}$, such that an edge $e$ of $K_{2 n}$ is colored under $\varphi^{\prime}$ if and only if $e$ is precolored under $\varphi$ or $e$ is a conflict edge of $h^{\prime}$ with $L$.

Lemma 5. Let $\alpha, \beta$ be constants and $c(n), f(n)$ be functions of $n$ such that

$$
m-\beta m-2 \alpha m-2 c(n)-\frac{2 n c(n)}{f(n)} \geqslant 1 .
$$

There is a proper $m$-edge precoloring $\varphi^{\prime}$ of $K_{2 n}$ satisfying the following:

(a) $\varphi^{\prime}(u v)=\varphi(u v)$ for any edge uv of $K_{2 n}$ that is precolored under $\varphi$.

(b) For every conflict edge uv of $h^{\prime}$ that is not colored under $\varphi$, uv is colored under $\varphi^{\prime}$ and $\varphi^{\prime}(u v) \notin L(u v)$.

(c) There are at most $\alpha m+c(n)$ prescribed edges at each vertex of $K_{2 n}$ under $\varphi^{\prime}$.

(d) There are at most $\alpha m+f(n)$ prescribed edges with color $i, i=1, \ldots, m$, under $\varphi^{\prime}$.

Furthermore, the edge coloring $h^{\prime}$ of $K_{2 n}$ and the precoloring $\varphi^{\prime}$ of $K_{2 n}$ satisfy that

(e) For each color $c \in\{1,2, \ldots, n\}$, there are at most $2 c(n)$ prescribed edges in $K_{n, n}$ with color $c$ under $h^{\prime}$.

(f) For each color $c \in\{n+1, n+2, \ldots, m\}$, there are at most $2 c(n)$ prescribed edges in $G_{1}\left(G_{2}\right)$ with color $c$ under $h^{\prime}$.

The proof of this lemma is almost identical to the proof of a similar claim for $K_{n, n}$ in Step III in [5]; thus we omit the details of this proof.

Using Lemma 5, from $\varphi$ we construct a coloring $\varphi^{\prime}$ satisfying the conditions in the lemma. Note that the two conditions (e) and (f) imply the following.

(g) For each color $c \in\{1,2, \ldots, m\}$, there are at most $2 c(n)$ prescribed edges in $K_{2 n}$ with color $c$ in $h^{\prime}$.

Step IV: Let $h^{\prime}$ be the $m$-edge coloring of $K_{2 n}$ obtained in Step II, and suppose that $\hat{h}$ is a proper $m$-edge coloring of $K_{2 n}$ obtained from $h^{\prime}$ by performing a sequence of swaps. We say that an edge $e$ in $K_{2 n}$ is disturbed (in $\hat{h}$ ) if $e$ appears in a swap which is used for obtaining $\hat{h}$ from $h^{\prime}$, or if $e$ is one of the original at most $3 n+7$ edges in $h^{\prime}$ that do not belong to at least $\left\lfloor\frac{n}{2}\right\rfloor-\epsilon n$ allowed strong 2-colored 4-cycles in $h^{\prime}$. For a constant $d>0$, we say that a vertex $v$ or color $c$ is $d$-overloaded if at least $d n$ edges which are incident to $v$ or colored $c$, respectively, are disturbed.

As mentioned in the outline, in Step IV we shall prove a number of lemmas. In all these lemmas, we shall from an edge coloring $h^{\prime \prime}$ of $K_{2 n}$, that have been obtained from $h^{\prime}$ by performing some swaps, construct a new coloring $h^{T}$ of $K_{2 n}$ by recoloring a subgraph $T$ of $K_{2 n}$. In every lemma in Step IV, the obtained coloring $h^{T}$ shall satisfy the following conditions, where $\left\{t_{1}, \ldots, t_{a}\right\}$ is a set of colors used in the coloring $h^{\prime \prime}$ : 
(a) no edge with a color in $\left\{t_{1}, \ldots, t_{a}\right\}$ appears in $T$;

(b) if there is a conflict of $h^{T}$ with respect to $L$, then this edge is also a conflict of $h^{\prime \prime}$;

(c) any edge in $G_{1}$ or $G_{2}$ that is requested under $h^{T}$ (with respect to $\varphi^{\prime}$ ) is also requested under $h^{\prime \prime}$.

For brevity, we say that a coloring $h^{T}$ obtained from $h^{\prime \prime}$ by recoloring a subgraph $T$ of $K_{2 n}$ as described above is good if it satisfies conditions (a)-(c).

The following lemma is similar to Lemmas 3.5 and 3.6 in [5], which are strengthened variants of Lemma 2.2 in [7]; thus, we shall skip the proof.

Lemma 6. Suppose that $h^{\prime \prime}$ is a proper m-edge coloring of $K_{2 n}$ obtained from $h^{\prime}$ by performing some sequence of swaps on $h^{\prime}$ and that at most $k n^{2}$ edges in $h^{\prime \prime}$ are disturbed for some constant $k>0$. Suppose that for each color $c$, at most $2 c(n)+P(n)$ edges with color c under $h^{\prime \prime}$ are prescribed. Moreover, let $\left\{t_{1}, \ldots, t_{a}\right\}$ be a set of colors from $h^{\prime \prime}$. If

$$
\left\lfloor\frac{n}{2}\right\rfloor-2 \epsilon n-6 d n-5 \frac{k}{d} n-4 \alpha m-8 c(n)-3 a-3 \beta m-2 P(n)-6>0
$$

then for any vertex $u_{1}$ of $G_{1}\left(G_{2}\right)$ and all but at most

- $2 \frac{k}{d} n+\alpha m+c(n)+a$ choices of a vertex $u_{2}$ in $G_{2}\left(G_{1}\right)$, such that $h^{\prime \prime}\left(u_{1} u_{2}\right) \in$ $\{1,2, \ldots, n\}$, and

- $4 \frac{k}{d} n+a+1+4 c(n)+2 \beta m+2 \alpha m+2 d n+P(n)$ choices of a vertex $v_{2}$ in $G_{2}\left(G_{1}\right)$, such that $h^{\prime \prime}\left(u_{1} v_{2}\right) \in\{1,2, \ldots, n\}$,

there is a subgraph $T$ of $K_{n, n}$ and a proper $m$-edge coloring $h^{T}$ of $K_{2 n}$, obtained from $h^{\prime \prime}$ by performing a sequence of swaps on 4 -cycles in $T$, that satisfies the following:

- the color of any edge of $T$ under $h^{\prime \prime}$ is not d-overloaded;

- no edges that are prescribed (with respect to $\varphi^{\prime}$ ) are in $T$;

- $h^{\prime \prime}$ and $h^{T}$ differs on at most 16 edges (i.e. T contains at most 16 edges);

- $h^{T}\left(u_{1} u_{2}\right)=h^{\prime \prime}\left(u_{1} v_{2}\right)$ and $h^{T}\left(u_{1} v_{2}\right)=h^{\prime \prime}\left(u_{1} u_{2}\right)$;

- $h^{T}$ is good.

Lemma 6 states that there are many pairs of adjacent edges $e_{x}, e_{y} \in E\left(K_{n, n}\right)$ satisfying that $h^{\prime \prime}\left(e_{x}\right), h^{\prime \prime}\left(e_{y}\right) \in\{1,2, \ldots, n\}$ such that we can exchange their colors by recoloring a small subgraph of $K_{n, n}$. When applying the preceding lemma, we shall refer to $u_{1} u_{2}$ as the "first edge" and $u_{1} v_{2}$ as the "second edge".

Given an edge $e_{x} \in E\left(K_{n, n}\right)$ such that $h^{\prime \prime}\left(e_{x}\right) \in\{1,2, \ldots, n\}$, the following lemma is used for obtaining a coloring where an edge $e_{y} \in E\left(K_{n, n}\right)$ adjacent to $e_{x}$ is colored $h^{\prime \prime}\left(e_{x}\right)$. 
Lemma 7. Suppose that $h^{\prime \prime}$ is a proper $m$-edge coloring of $K_{2 n}$ obtained from $h^{\prime}$ by performing some sequence of swaps on $h^{\prime}$ and that at most $k n^{2}$ edges in $h^{\prime \prime}$ are disturbed for some constant $k>0$. Suppose that for each color $c$, at most $2 c(n)+P(n)$ edges with color $c$ under $h^{\prime \prime}$ are prescribed, and at most $H(n)$ edges with color $c$ are disturbed. Moreover, let $\left\{t_{1}, \ldots, t_{a}\right\}$ be a set of colors from $h^{\prime \prime}$. If

$$
\left\lfloor\frac{n}{2}\right\rfloor-2 \epsilon n-6 d n-5 \frac{k+34 / n^{2}}{d} n-4 \alpha m-8 c(n)-3 a-3 \beta m-2 P(n)-6>0
$$

and

$$
n-\left(8 \frac{k+34 / n^{2}}{d} n+2 a+3+8 c(n)+6 \beta m+4 \alpha m+4 d n+2 P(n)+H(n)\right)>0
$$

then for any edge $u_{1} u_{2}$ of $K_{n, n}$ with

$$
h^{\prime \prime}\left(u_{1} u_{2}\right)=c_{1}, c_{1} \in\{1,2, \ldots, n\}, c_{1} \notin\left\{t_{1}, \ldots, t_{a}\right\}
$$

and all but at most

$$
4 c(n)+P(n)+2 \beta m+2 \alpha m+2 a+1+4 \frac{k+34 / n^{2}}{d} n+H(n)
$$

choices of a vertex $v_{2}$ satisfying that $u_{1} v_{2} \in E\left(K_{n, n}\right)$, there is a subgraph $T$ of $K_{n, n}$ and a proper $m$-edge coloring $h^{T}$ of $K_{2 n}$, obtained from $h^{\prime \prime}$ by performing a sequence of swaps on 4-cycles in $T$, that satisfies the following:

- except $c_{1}$, any color of an edge in $T$ under $h^{\prime \prime}$ is not d-overloaded;

- except $u_{1} u_{2}$, no edge in $T$ is prescribed;

- $h^{\prime \prime}$ and $h^{T}$ differs on at most 34 edges (i.e. T contains at most 34 edges);

- $h^{T}\left(u_{1} v_{2}\right)=h^{\prime \prime}\left(u_{1} u_{2}\right)=c_{1}$;

- $h^{T}$ is good.

Proof. Without loss of generality, assume that $u_{1} \in V\left(G_{1}\right)$; this implies $u_{2} \in V\left(G_{2}\right)$. We choose $v_{1} \in V\left(G_{1}\right)$ and $v_{2} \in V\left(G_{2}\right)$ so that the following properties hold.

- The edge $v_{1} v_{2}$ in $K_{n, n}$ satisfying $h^{\prime \prime}\left(v_{1} v_{2}\right)=c_{1}$ is not disturbed and not prescribed. Since there are at most $2 c(n)+P(n)$ prescribed edges and at most $H(n)$ disturbed edges with color $c_{1}$ under $h^{\prime \prime}$, and each such prescribed or disturbed edge of $K_{n, n}$ can be incident to at most one vertex of $G_{2}$, this eliminates at most $2 c(n)+P(n)+H(n)$ choices.

- The edge $u_{1} v_{2}$ and the edge $u_{2} v_{1}$ are both valid choices for the first edge in an application of Lemma 6 . This eliminates at most

$$
2\left(2 \frac{k+34 / n^{2}}{d} n+\alpha m+c(n)+a\right)
$$

choices. The additive factor $34 / n^{2}$ comes from the fact that Lemma 6 is applied twice when performing a sequence of swaps to transform $h^{\prime \prime}$ into $h^{T}$. 
- $c_{1} \notin L\left(u_{1} v_{2}\right) \cup L\left(u_{2} v_{1}\right)$ and $u_{1} u_{2} \neq v_{1} v_{2}$. This excludes at most $2 \beta m+1$ choices.

Thus we have at least

$$
n-4 c(n)-P(n)-2 \beta m-2 \alpha m-2 a-1-4 \frac{k+34 / n^{2}}{d} n-H(n)
$$

choices for a vertex $v_{2}$ and an edge $v_{1} v_{2}$. We note that this expression is greater than zero by assumption, so we can indeed make the choice.

Next, we want to choose a color $c_{2} \in\{1,2, \ldots, n\}$ such that the following properties hold.

- The edges $e_{1}$ and $e_{2}$ colored $c_{2}$ under $h^{\prime \prime}$ that are incident with $u_{1}$ and $u_{2}$, respectively, are both valid choices for the second edge in an application of Lemma 6; this eliminates at most

$$
2\left(4 \frac{k+34 / n^{2}}{d} n+a+1+4 c(n)+2 \beta m+2 \alpha m+2 d n+P(n)\right)
$$

choices. Note that this condition implies that color $c_{2}$ is not $d$-overloaded.

- $c_{2} \neq c_{1}$ and $c_{2} \notin L\left(u_{1} u_{2}\right) \cup L\left(v_{1} v_{2}\right)$. This excludes at most $2 \beta m+1$ choices.

Thus we have at least

$$
n-\left(8 \frac{k+34 / n^{2}}{d} n+2 a+3+8 c(n)+6 \beta m+4 \alpha m+4 d n+2 P(n)\right)
$$

choices. By assumption, this expression is greater than zero, so we can indeed choose such color $c_{2}$. Now, since

$$
\left\lfloor\frac{n}{2}\right\rfloor-2 \epsilon n-6 d n-5 \frac{k+34 / n^{2}}{d} n-4 \alpha m-8 c(n)-3 a-3 \beta m-2 P(n)-6>0,
$$

we can apply Lemma 6 two consecutive times to exchange the color of $u_{1} v_{2}$ and $e_{1}$, and similarly for $u_{2} v_{1}$ and $e_{2}$. Finally, by swapping on the 2-colored 4-cycle $u_{1} u_{2} v_{1} v_{2} u_{1}$, we get the proper coloring $h^{T}$ such that $h^{T}\left(u_{1} v_{2}\right)=h^{\prime \prime}\left(u_{1} u_{2}\right)=c_{1}$.

Note that the subgraph $T$, consisting of all edges used in the swaps above, contains two edges $u_{1} u_{2}$ and $v_{1} v_{2}$ and the additional edges needed for two applications of Lemma 6 ; this implies that $T$ contains at most $2+16 \times 2=34$ edges. Furthermore, except (possibly) $u_{1} u_{2}$, no edges in $T$ are prescribed; except $c_{1}, T$ only contains edges with colors that are not $d$-overloaded.

Since the applications of Lemma 6 do not result in any "new" requested edges in $G_{1}$ or $G_{2}$, the transformations in this lemma do not yield any "new" requested edges in $G_{1}$ or $G_{2}$; the same holds for conflict edges in $K_{2 n}$. Additionally, $T$ does not contain an edge with a color in $\left\{t_{1}, \ldots, t_{a}\right\}$. Thus $h^{T}$ is good. 
As for Lemma 6, when applying Lemma 7, we shall refer to $u_{1} u_{2}$ as the "first edge" and $u_{1} v_{2}$ as the "second edge".

We use Lemma 8 below for transforming a coloring $h^{\prime \prime}$ into a coloring where an edge $e_{y} \in E\left(K_{n, n}\right)$ is colored by the color $h^{\prime \prime}\left(e_{x}\right)$ of an adjacent edge $e_{x} \in E\left(G_{1}\right)\left(E\left(G_{2}\right)\right)$, where $h^{\prime \prime}\left(e_{x}\right) \in\{n+1, \ldots, m\}$. In applications of this lemma $u_{1} v_{1}$ will be referred to as the "first edge", and $u_{1} u_{2}$ as the "second edge".

Lemma 8. Suppose that $h^{\prime \prime}$ is a proper $m$-edge coloring of $K_{2 n}$ obtained from $h^{\prime}$ by performing some sequence of swaps on $h^{\prime}$ and that at most $k n^{2}$ edges in $h^{\prime \prime}$ are disturbed for some constant $k>0$. Suppose further that for each color $c$, at most $2 c(n)+P(n)$ edges with color $c$ under $h^{\prime \prime}$ are prescribed, and at most $H(n)$ edges with color $c$ are disturbed. Moreover, let $\left\{t_{1}, \ldots, t_{a}\right\}$ be a set of colors from $h^{\prime \prime}$. If

$$
\left\lfloor\frac{n}{2}\right\rfloor-2 \epsilon n-6 d n-5 \frac{k+34 / n^{2}}{d} n-4 \alpha m-8 c(n)-3 a-3 \beta m-2 P(n)-6>0
$$

and

$$
n-\left(8 \frac{k+34 / n^{2}}{d} n+2 a+2+12 c(n)+6 \beta m+8 \alpha m+4 d n+2 P(n)+2 H(n)\right)>0
$$

then for any edge $u_{1} v_{1}$ of $G_{1}\left(G_{2}\right)$ with

$$
h^{\prime \prime}\left(u_{1} v_{1}\right)=c_{1}, c_{1} \in\{n+1, \ldots, m\}, c_{1} \notin\left\{t_{1}, \ldots, t_{a}\right\}
$$

and all but at most

$$
6 c(n)+2 P(n)+2 \beta m+2 \alpha m+2 a+1+4 \frac{k+34 / n^{2}}{d} n+2 H(n)
$$

choices of $u_{2} \in V\left(G_{2}\right)\left(V\left(G_{1}\right)\right)$, there is a subgraph $T$ of $K_{2 n}$ and a proper $m$-edge coloring $h^{T}$, obtained from $h^{\prime \prime}$ by performing a sequence of swaps on 4-cycles in $T$, that satisfies the following:

- except $c_{1}$, any color of an edge in $T$ under $h^{\prime \prime}$ is not d-overloaded;

- except $u_{1} v_{1}$, no edge in $T$ is prescribed;

- $h^{\prime \prime}$ and $h^{T}$ differs on at most 34 edges (i.e. T contains at most 34 edges);

- $h^{T}\left(u_{1} u_{2}\right)=h^{\prime \prime}\left(u_{1} v_{1}\right)=c_{1}$;

- $h^{T}$ is good.

Proof. Without loss of generality, assume that $u_{1} v_{1} \in E\left(G_{1}\right)$. We choose the vertices $u_{2}, v_{2} \in V\left(G_{2}\right)$ such that the following properties hold.

- The edge $u_{2} v_{2} \in E\left(G_{2}\right)$ satisfying $h^{\prime \prime}\left(u_{2} v_{2}\right)=c_{1}$ is not disturbed and not prescibed. Since there are at most $2 c(n)+P(n)$ prescribed edges and at most $H(n)$ disturbed edges with color $c_{1}$ under $h^{\prime \prime}$; and each prescribed or disturbed edge of $G_{2}$ can be incident to at most two vertices of $G_{2}$, this eliminates at most $2(2 c(n)+P(n)+H(n))$ choices. 
- The edges $u_{1} u_{2}$ and $v_{1} v_{2}$ are both valid choices for the first edge in an application of Lemma 6. As in the proof of the preceding lemma, this eliminates at most

$$
2\left(2 \frac{k+34 / n^{2}}{d} n+\alpha m+c(n)+a\right)
$$

choices.

- $c_{1} \notin L\left(u_{1} u_{2}\right) \cup L\left(v_{1} v_{2}\right)$. This excludes at most $2 \beta m$ choices.

In the coloring $h^{\prime}$, there are at least $n-1$ vertices in $G_{2}$ that are incident with an edge of color $c_{1}$; thus we have at least

$$
n-1-6 c(n)-2 P(n)-2 \beta m-2 \alpha m-2 a-4 \frac{k+34 / n^{2}}{d} n-2 H(n)
$$

choices for $u_{2}$. We note that this expression is greater than zero by assumption, so we can indeed make the choice.

Next, we want to choose a color $c_{2} \in\{1,2, \ldots, n\}$ (which implies $c_{2} \neq c_{1}$ ) such that the following properties hold.

- The edges $e_{1}$ and $e_{2}$ colored $c_{2}$ under $h^{\prime \prime}$ that are incident with $u_{1}$ and $v_{1}$, respectively, are both valid choices for the second edge in an application of Lemma 6; this eliminates at most

$$
2\left(4 \frac{k+34 / n^{2}}{d} n+a+1+4 c(n)+2 \beta m+2 \alpha m+2 d n+P(n)\right)
$$

choices.

- $c_{2} \notin L\left(u_{1} v_{1}\right) \cup L\left(u_{2} v_{2}\right)$. This excludes at most $2 \beta m$ choices.

- $c_{2} \notin \varphi^{\prime}\left(u_{1}\right) \cup \varphi^{\prime}\left(u_{2}\right) \cup \varphi^{\prime}\left(v_{1}\right) \cup \varphi^{\prime}\left(v_{2}\right) \backslash\left\{\varphi^{\prime}\left(u_{1} v_{1}\right), \varphi^{\prime}\left(u_{2} v_{2}\right)\right\}$. This condition is needed to ensure that performing a series of swaps on $T$, does not result in a "new" requested edge in $G_{1}$ or $G_{2}$. Since there are at most $\alpha m+c(n)$ prescribed edges at each vertex of $K_{2 n}$ under $\varphi^{\prime}$, this excludes at most $4(\alpha m+c(n))$ choices.

Thus we have at least

$$
n-\left(8 \frac{k+34 / n^{2}}{d} n+2 a+2+12 c(n)+6 \beta m+8 \alpha m+4 d n+2 P(n)\right)
$$

choices. By assumption, this expression is greater than zero, so we can indeed choose such color $c_{2}$. Now, since

$$
\left\lfloor\frac{n}{2}\right\rfloor-2 \epsilon n-6 d n-5 \frac{k+34 / n^{2}}{d} n-4 \alpha m-8 c(n)-3 a-3 \beta m-2 P(n)-6>0,
$$

we can apply Lemma 6 two consecutive times to exchange the colors of $u_{1} u_{2}$ and $e_{1}$, and similarly for $v_{1} v_{2}$ and $e_{2}$. Finally, by swapping on the 2-colored 4-cycle $u_{1} u_{2} v_{2} v_{1} u_{1}$, we get the proper coloring $h^{T}$ such that $h^{T}\left(u_{1} u_{2}\right)=h^{\prime \prime}\left(u_{1} v_{1}\right)=c_{1}$. 
Note that the subgraph $T$, consisting of all edges used in the swaps above, contains two edges $u_{1} v_{1}$ and $u_{2} v_{2}$ and the additional edges needed for two applications of Lemma 6 ; this implies that $T$ uses at most $2+16 \times 2=34$ edges. Furthermore, except (possibly) $u_{1} v_{1}$, no edges in $T$ are prescribed; except $c_{1}, T$ only contains edges with colors that are not $d$-overloaded.

Finally, as in the proof of the preceding Lemma, since the applications of Lemma 6 result in a good coloring of $K_{2 n}$, the coloring $h^{T}$ is good.

The following lemma is used for transforming a coloring $h^{\prime \prime}$ into a coloring where an edge $e_{y} \in E\left(K_{n, n}\right)$ is colored by the color $h^{\prime \prime}\left(e_{x}\right)$ of an adjacent edge $e_{x} \in E\left(K_{n, n}\right)$, where $h^{\prime \prime}\left(e_{x}\right) \in\{n+1, \ldots, m\}$. When applying the lemma we shall refer to $u_{1} u_{2}$ as the "first edge" and $u_{1} v_{2}$ as the "second edge".

Lemma 9. Suppose that $h^{\prime \prime}$ is a proper $m$-edge coloring of $K_{2 n}$ obtained from $h^{\prime}$ by performing some sequence of swaps on $h^{\prime}$ and that at most $k n^{2}$ edges in $h^{\prime \prime}$ are disturbed for some constant $k>0$. Suppose further that for each color $c$, at most $2 c(n)+P(n)$ edges with color $c$ under $h^{\prime \prime}$ are prescribed, and at most $H(n)$ edges with color $c$ are disturbed. Let $\left\{t_{1}, \ldots, t_{a}\right\}$ be a set of colors from $h^{\prime \prime}$. If

$$
\left\lfloor\frac{n}{2}\right\rfloor-2 \epsilon n-6 d n-5 \frac{k+101 / n^{2}}{d} n-4 \alpha m-8 c(n)-3 a-3 \beta m-2 P(n)-6>0
$$

and

$$
n-\left(8 \frac{k+101 / n^{2}}{d} n+2 a+2+12 c(n)+6 \beta m+8 \alpha m+4 d n+2 P(n)+2 H(n)\right)>0
$$

then for any edge $u_{1} u_{2}$ of $K_{n, n}$ with

$$
h^{\prime \prime}\left(u_{1} u_{2}\right)=c_{1}, c_{1} \in\{n+1, \ldots, m\}, c_{1} \notin\left\{t_{1}, \ldots, t_{a}\right\}
$$

and all but at most

$$
5 c(n)+2 P(n)+\alpha m+\beta m+a+2+2 \frac{k+67 / n^{2}}{d} n+2 H(n)
$$

choices of a vertex $v_{2}$ satisfying $u_{1} v_{2} \in K_{n, n}$, there is a subgraph $T$ of $K_{2 n}$ and a proper $m$-edge coloring $h^{T}$, obtained from $h^{\prime \prime}$ by performing a sequence of swaps on 4-cycles in $T$, that satisfies the following:

- except $c_{1}$, any color of an edge in $T$ under $h^{\prime \prime}$ is not d-overloaded;

- except $u_{1} u_{2}$, no edge of $T$ is prescribed;

- $h^{\prime \prime}$ and $h^{T}$ differs on at most 67 edges (i.e. T contains at most 67 edges);

- $h^{T}\left(u_{1} v_{2}\right)=h^{\prime \prime}\left(u_{1} u_{2}\right)=c_{1}$;

- $h^{T}$ is good. 
Proof. Without loss of generality, assume that $u_{1} \in V\left(G_{1}\right)$; this implies $u_{2} \in V\left(G_{2}\right)$. We choose the vertices $v_{2}, x \in V\left(G_{2}\right)$ such that the following properties hold.

- The edge $v_{2} x \in E\left(G_{2}\right)$ satisfying $h^{\prime \prime}\left(v_{2} x\right)=c_{1}$ is not disturbed. As in the proof of the preceding lemma, this eliminates at most $2 H(n)$ choices.

- The edge $v_{2} x$ is not prescribed and $v_{2} \neq u_{2}$. This eliminates at most $2(2 c(n)+$ $P(n))+1$ choices.

- The edge $u_{1} v_{2}$ is a valid choice for the first edge in an application of Lemma 6 . This eliminates at most $2 \frac{k+67 / n^{2}}{d} n+\alpha m+c(n)+a$ choices.

- $L\left(u_{1} v_{2}\right)$ does not contain the color $c_{1}$. This eliminates at most $\beta m$ choices.

In the coloring $h^{\prime}$, there are at least $n-1$ vertices in $G_{2}$ that are incident with an edge of color $c_{1}$; thus we have at least

$$
n-1-5 c(n)-2 P(n)-\alpha m-\beta m-a-1-2 \frac{k+67 / n^{2}}{d} n-2 H(n)
$$

choices for $v_{2}$. Since this expression is greater than zero by assumption, we can indeed make the choice.

Next, we want to choose a vertex $v_{1} \in V\left(G_{1}\right)$ satisfying the following:

- The edge $v_{2} v_{1}$ is a valid choice for the second edge in an application of Lemma 8 . This eliminates at most

$$
6 c(n)+2 P(n)+2 \beta m+2 \alpha m+2 a+1+4 \frac{k+(34+67) / n^{2}}{d} n+2 H(n)
$$

choices.

- The edge $u_{2} v_{1}$ is a valid choice for the first edge in an application of Lemma 6 and $v_{1} \neq u_{1}$. This eliminates at most $2 \frac{k+67 / n^{2}}{d} n+\alpha m+c(n)+a+1$ choices.

- $L\left(u_{2} v_{1}\right)$ does not contain the color $c_{1}$. This eliminates at most $\beta m$ choices.

Thus we have at least

$$
n-7 c(n)-2 P(n)-3 \alpha m-3 \beta m-3 a-2-6 \frac{k+101 / n^{2}}{d} n-2 H(n)
$$

choices for $v_{1}$. Since this expression is greater than zero by assumption, we can indeed make the choice.

Finally, we want to choose a color $c_{2} \in\{1,2, \ldots, n\}$ (which implies $c_{2} \neq c_{1}$ ) such that the following properties hold. 
- The edges $e_{1}$ and $e_{2}$ colored $c_{2}$ under $h^{\prime \prime}$ that are adjacent to $u_{1}$ and $u_{2}$, respectively, are both valid choices for the second edge in an application of Lemma 6 ; this eliminates at most

$$
2\left(4 \frac{k+67 / n^{2}}{d} n+a+1+4 c(n)+2 \beta m+2 \alpha m+2 d n+P(n)\right)
$$

choices.

- $c_{2} \notin L\left(u_{1} u_{2}\right) \cup L\left(v_{1} v_{2}\right)$. This excludes at most $2 \beta m$ choices.

Thus we have at least

$$
n-\left(8 \frac{k+67 / n^{2}}{d} n+2 a+2+8 c(n)+6 \beta m+4 \alpha m+4 d n+2 P(n)\right)
$$

choices. By assumption, this expression is greater than zero, so we can indeed choose such edges $e_{1}$ and $e_{2}$.

Now, since

$$
\left\lfloor\frac{n}{2}\right\rfloor-2 \epsilon n-6 d n-5 \frac{k+101 / n^{2}}{d} n-4 \alpha m-8 c(n)-3 a-3 \beta m-2 P(n)-6>0
$$

and

$$
n-\left(8 \frac{k+101 / n^{2}}{d} n+2 a+2+12 c(n)+6 \beta m+8 \alpha m+4 d n+2 P(n)+2 H(n)\right)>0,
$$

we can apply Lemma 6 two consecutive times to exchange the colors of $u_{1} v_{2}$ and $e_{1}$, and similarly for $u_{2} v_{1}$ and $e_{2}$. We can thereafter apply Lemma 8 to obtaing a coloring where $v_{1} v_{2}$ is colored $c_{1}$. Now, by swapping on the 2 -colored 4 -cycle $u_{1} u_{2} v_{1} v_{2} u_{1}$, we get the proper coloring $h^{T}$ such that $h^{T}\left(u_{1} v_{2}\right)=h^{\prime \prime}\left(u_{1} u_{2}\right)=c_{1}$.

Note that the subgraph $T$, consisting of all edges used in the swaps above contains an edge $u_{1} u_{2}$ and all the additional edges needed for applying Lemma 6 twice and Lemma 8 once; this implies that $T$ contains at most $1+16 \times 2+34=67$ edges. Furthermore, except $u_{1} u_{2}$, no edges in $T$ are prescribed; except $c_{1}, T$ only contains edges with colors that are not $d$-overloaded.

Finally, since the applications of Lemma 6 and 8 do not result in any "new" requested edges in $G_{1}$ or $G_{2}$, the transformations in this lemma do not yield any "new" requested edges in $G_{1}$ or $G_{2}$; the same holds for conflict edges in $K_{2 n}$. Additionally, $T$ does not contain an edge with a color in $\left\{t_{1}, \ldots, t_{a}\right\}$, so in conclusion, $h^{T}$ is good.

Given a color $c_{1} \in\{1,2, \ldots, n\}$, the final lemma in this step is used for obtaining a coloring where an edge in $G_{1}$ or $G_{2}$ is colored $c_{1}$. In applications of this lemma we shall refer to $u v$ as the "first edge".

Lemma 10. Suppose that $h^{\prime \prime}$ is a proper $m$-edge coloring of $K_{2 n}$ obtained from $h^{\prime}$ by performing some sequence of swaps on $h^{\prime}$ and that at most $k n^{2}$ edges in $h^{\prime \prime}$ are disturbed for some constant $k>0$. Suppose further that for each color $c$, at most $2 c(n)+P(n)$ edges 
with color $c$ under $h^{\prime \prime}$ are prescribed, and at most $H(n)$ edges with color $c$ are disturbed. Moreover, let $\left\{t_{1}, \ldots, t_{a}\right\}$ be a set of colors from $h^{\prime \prime}$. If

$$
\left\lfloor\frac{n}{2}\right\rfloor-2 \epsilon n-6 d n-8 \frac{k+104 / n^{2}}{d} n-4 \alpha m-15 c(n)-4 a-6 \beta m-5 P(n)-2 H(n)-6>0
$$

then for any color $c_{1} \in\{1,2, \ldots, n\}$, where $c_{1} \notin\left\{t_{1}, \ldots, t_{a}\right\}$, there are at least

$$
\left\lfloor\frac{n}{2}\right\rfloor-7 c(n)-3 P(n)-d n-2 H(n)
$$

choices of an edge uv $\in E\left(G_{1}\right)\left(E\left(G_{2}\right)\right)$, such that there is a subgraph $T$ of $K_{2 n}$ and a proper $m$-edge coloring $h^{T}$, obtained from $h^{\prime \prime}$ by performing a sequence of swaps on 4-cycles in $T$, that satisfies the following:

- except $c_{1}$, any color of an edge in $T$ under $h^{\prime \prime}$ is not d-overloaded;

- $T$ contains no prescribed edge;

- $h^{\prime \prime}$ and $h^{T}$ differs on at most 70 edges (i.e. T contains at most 70 edges);

- $h^{T}(u v)=c_{1}$;

- $h^{T}$ is good.

Proof. We will prove the lemma assuming $u v \in E\left(G_{1}\right)$; the case when $u v \in E\left(G_{2}\right)$ is of course analogous. Since at most $k n^{2}$ edges in $h^{\prime \prime}$ are disturbed, there are at most $k n / d$ $d$-overloaded colors; by assumption, $n-1-k n / d-a>0$, so we can choose a color $c_{2} \in\{n+1, n+2, \ldots, m\}$ such that $c_{2} \notin\left\{t_{1}, \ldots, t_{a}\right\}$ is not a $d$-overloaded color. Next, we choose an edge $u v \in E\left(G_{1}\right)$ satisfying $h^{\prime \prime}(u v)=c_{2}$ such that the following properties hold.

- The edge $u v$ is not prescribed. Since there are at most $2 c(n)+P(n)$ prescribed edges with color $c_{2}$ in $h^{\prime \prime}$, this eliminates at most $2 c(n)+P(n)$ choices.

- The edge $u v$ is not disturbed and $c_{1} \notin L(u v)$. Since the color $c_{2}$ is not $d$-overloaded and for each pair of colors $c_{1}, c_{2} \in\{1,2, \ldots, m\}$, there are at most $c(n)$ edges $e$ in $K_{2 n}$ with $h^{\prime}(e)=c_{2}$ and $c_{1} \in L(e)$ and at most $d n$ edges of color $c_{2}$ have been used in the swaps for transforming $h^{\prime}$ to $h^{\prime \prime}$; this eliminates at most $c(n)+d n$ choices.

- $c_{1} \notin \varphi^{\prime}(u) \cup \varphi^{\prime}(v) \backslash\left\{\varphi^{\prime}(u v)\right\}$. This condition is needed to ensure that after performing the swaps in this lemma, $u v$ is not a requested edge in $G_{1}$. Since there are at most $2 c(n)+P(n)$ prescribed edges with color $c_{1}$ in $h^{\prime \prime}$, this excludes at most $2(2 c(n)+$ $P(n))$ choices.

- The edges $e_{1}$ and $e_{2}$ colored $c_{1}$ under $h^{\prime \prime}$ that are incident with $u$ and $v$, respectively, are not disturbed. This condition implies that $e_{1}, e_{2} \in K_{n, n}$ and this eliminates at most $2 H(n)$ choices. 
Under $h^{\prime}$ there are $\left\lfloor\frac{n}{2}\right\rfloor$ edges in $G_{2}$ that are colored $c_{2}$; thus we have at least

$$
\left\lfloor\frac{n}{2}\right\rfloor-7 c(n)-3 P(n)-d n-2 H(n)
$$

choices for an edge $u v$. Since this expression is greater than zero by assumption, we can indeed make the choice.

Next, we want to choose an edge $x y \in E\left(G_{2}\right)$ satisfying $h^{\prime \prime}(x y)=c_{2}$ such that the following properties hold.

- $c_{1} \notin L(x y) \cup \varphi^{\prime}(x) \cup \varphi^{\prime}(y) \backslash\left\{\varphi^{\prime}(x y)\right\}$, and the edge $x y$ is not prescibed and not disturbed. As before, this eliminates at most $7 c(n)+3 P(n)+d n$ choices.

- The edges $u x$ and $v y$ are both valid choices for the second edge in an application of Lemma 7. This eliminates at most

$$
2\left(4 c(n)+P(n)+2 \beta m+2 \alpha m+2 a+1+4 \frac{k+(34+70) / n^{2}}{d} n+H(n)\right)
$$

choices.

- $c_{2} \notin L(u x) \cup L(v y)$. This eliminates at most $2 \beta m$ choices.

Thus we have at least

$$
\left\lfloor\frac{n}{2}\right\rfloor-\left(15 c(n)+5 P(n)+4 \alpha m+6 \beta m+d n+4 a+2+8 \frac{k+104 / n^{2}}{d} n+2 H(n)\right)
$$

choices for $x y$. Since this expression is greater than zero by assumption, we can indeed make the choice.

Now, since

$$
\left\lfloor\frac{n}{2}\right\rfloor-2 \epsilon n-6 d n-8 \frac{k+104 / n^{2}}{d} n-4 \alpha m-15 c(n)-4 a-6 \beta m-5 P(n)-2 H(n)-6>0
$$

we can apply Lemma 7 two consecutive times to obtain a coloring where $u x$ is colored $c_{1}$ and $v u$ is colored $c_{1}$. Thereafter, finally, by swapping on the 2-colored 4-cycle uvyxu, we get the proper coloring $h^{T}$ such that $h^{T}(u v)=h^{\prime \prime}\left(e_{1}\right)=c_{1}$.

Note that the subgraph $T$, consisting of all edges used in the swaps above, contains two edges $u v$ and $x y$ and all additional edges needed for applying Lemma 7 twice; this implies that $T$ contains at most $2+34 \times 2=70$ edges. Furthermore, none of these edges in $T$ are prescribed; except $c_{1}, T$ only contains edges with colors that are not $d$-overloaded.

Finally, let us note that since the applications of Lemma 7 results in good edge colorings of $K_{2 n}$, the coloring $h^{T}$ is good.

Step V: Let $\varphi^{\prime}$ be the proper $m$-precoloring of $K_{2 n}$ obtained in Step III and $h^{\prime}$ be the $m$-edge coloring of $K_{2 n}$ obtained in Step II. In this step we shall from $h^{\prime}$ construct a coloring $h_{q}$ of $K_{2 n}$ that agrees with $\varphi$ and which avoids $L$. This is done iteratively by steps: in each step we consider a prescribed edge $e$ of $K_{2 n}$, such that $h^{\prime}(e) \neq \varphi^{\prime}(e)$, 
and perform a sequence of swaps on 2-colored 4-cycles to obtain a coloring $h_{e}$ where $e$ is colored $\varphi^{\prime}(e)$. In this process, special care is taken so that these swaps do not result in any new requested edges in $G_{1}$ or $G_{2}$; in particular, this implies that every requested edge with a color in $\{1,2, \ldots, n\}$ is always in $K_{n, n}$ for any intermediate coloring of $K_{2 n}$ that is constructed in this iterative procedure.

We shall use four different recoloring techniques in this step; these are described in the proof of the following lemma.

Lemma 11. Suppose that $h^{\prime \prime}$ is a proper $m$-edge coloring of $K_{2 n}$ obtained from $h^{\prime}$ by performing some sequence of swaps on $h^{\prime}$ and that at most $k n^{2}$ edges in $h^{\prime \prime}$ are disturbed for some constant $k>0$. Suppose further that

- for each color $c$, at most $2 c(n)+P(n)$ edges with color $c$ under $h^{\prime \prime}$ are prescribed;

- at most $H(n)$ edges with color c are disturbed;

- all requested edges with a color from $\{1,2, \ldots, n\}$ under $h^{\prime \prime}$ are in $K_{n, n}$.

- if $e$ is a prescribed edge of $K_{n, n}$ that satisfies $\varphi^{\prime}(e) \neq h^{\prime \prime}(e)$, then $h^{\prime \prime}(e) \in\{1, \ldots, n\}$.

Let uv be an edge of $K_{2 n}$ such that

$$
h^{\prime \prime}(u v)=c_{1}, \quad \varphi^{\prime}(u v)=c_{2}, \quad c_{1} \neq c_{2} .
$$

and set

$M=\left\lfloor\frac{n}{2}\right\rfloor-\left(2 \epsilon n+24 c(n)+6 d n+9 P(n)+6 \beta m+4 \alpha m+10+8 \frac{k+(67+205) / n^{2}}{d} n+6 H(n)\right)$

If $M>0$, then there is a subgraph $T$ of $K_{2 n}$ and a proper $m$-edge coloring $h^{T}$, obtained from $h^{\prime \prime}$ by performing a sequence of swaps on 4-cycles in $T$, that satisfies the following:

- $h^{T}(u v)=c_{2}$;

- $h^{\prime \prime}$ and $h^{T}$ differs on at most 205 edges (i.e. T contains at most 205 edges);

- besides uv, $h^{\prime \prime}$ and $h^{T}$ disagree on at most 2 prescribed edges;

- if $h^{\prime \prime}$ and $h^{T}$ disagree on a prescribed edge ab (where $\left.a b \neq u v\right)$, then ab is a requested edge, $h^{T}(a b)$ is not d-overloaded and $h^{\prime \prime}(a b) \neq \varphi^{\prime}(a b)$;

- the subgraph $T$ contains at most three edges with color $c_{1}$ under $h^{\prime \prime}$, and at most four edges with color $c_{2}$ under $h^{\prime \prime}$;

- except $c_{1}$ and $c_{2}$, no colors of edges in $T$ (under $h^{\prime \prime}$ ) are d-overloaded;

- if there is a conflict of $h^{T}$ with $L$, then this edge is also a conflict of $h^{\prime \prime}$ with $L$;

- any edge in $G_{1}$ or $G_{2}$ that is requested under $h^{T}$ (with respect to $\varphi^{\prime}$ ) is also requested under $h^{\prime \prime}$. 
Proof. We shall contruct a subgraph $T$ of $K_{2 n}$, and by performing a sequence of swaps on 4-cycles of $T$, we shall obtain the coloring $h^{T}$ from $h^{\prime \prime}$, where $h^{T}$ and $\varphi^{\prime}$ agree on the edge $u v$. We will accomplish this by applying Lemmas 6-10, and in our application of these lemmas, we will avoid the colors $\left\{c_{1}, c_{2}\right\}$; so $a=2$.

Let $e_{1}$ and $e_{2}$ be the requested edges incident with $u$ and $v$, respectively, satisfying that $h^{\prime \prime}\left(e_{1}\right)=h^{\prime \prime}\left(e_{2}\right)=c_{2}$.

We shall consider four different cases.

Case 1. $u v \in E\left(K_{n, n}\right)$ and $c_{2} \in\{1,2, \ldots, n\}$ :

Since under $h^{\prime \prime}$, all requested edges with colors in $\{1,2, \ldots, n\}$ are in $K_{n, n}, e_{1}, e_{2} \in$ $E\left(K_{n, n}\right)$. Moreover, by assumption $c_{2} \in\{1, \ldots, n\}$, so we can proceed as in the proof of Lemma 3.7 in [5] and use swaps on 4-cycles, all edges of which are contained in $K_{n, n}$, to obtain a coloring $h^{T}$ where $h^{T}(u v)=c_{2}$. Note also that this implies that every precolored edge $e$ of $K_{n, n}$ that satisfies $h^{\prime \prime}(e) \in\{1, \ldots, n\}$, also satisfies $h^{T}(e) \in\{1, \ldots, n\}$.

The swaps needed for obtaining the required coloring will involve at most 69 edges, as described in proof of Lemma 3.7 in [5]. The exact details of the transformation of the coloring $h^{\prime \prime}$ into $h^{T}$ are given in [5], so we omit them here.

Case 2. $u v \in E\left(K_{n, n}\right)$ and $c_{2} \in\{n+1, n+2, \ldots, m\}$ :

In this case, we will contruct a subgraph $T$ with at most 136 edges. Without loss of generality, we assume that $u \in V\left(G_{1}\right)$, this implies $v \in V\left(G_{2}\right)$. By assumption $c_{1} \in$ $\{1, \ldots, n\}$; we choose an edge $x y \in E\left(K_{n, n}\right)\left(x \in V\left(G_{1}\right)\right.$ and $\left.y \in V\left(G_{2}\right)\right)$, with $h^{\prime \prime}(x y)=c_{1}$ such that the following properties hold.

- The edge $x y$ is not disturbed and not prescribed and $c_{2} \notin L(x y)$. Since for each pair of colors $c_{1}, c_{2} \in\{1,2, \ldots, m\}$, there are at most $c(n)$ edges $e$ in $K_{2 n}$ with $h^{\prime}(e)=c_{1}$ and $c_{2} \in L(e)$, and at most $H(n)$ edges of color $c_{1}$ have been used in the swaps for transforming $h^{\prime}$ into $h^{\prime \prime}$, this eliminates at most $H(n)+2 c(n)+P(n)+c(n)$ choices.

- The vertex $x$ satisfies the following.

- If $e_{2} \in E\left(G_{2}\right)$, then we choose $x$ such that $v x$ is a valid choice for the second edge in an application of Lemma 8 . This eliminates at most

$$
6 c(n)+2 P(n)+2 \beta m+2 \alpha m+5+4 \frac{k+(34+136) / n^{2}}{d} n+2 H(n)
$$

choices.

- If $e_{2} \in E\left(K_{n, n}\right)$, then since $h^{\prime \prime}\left(e_{2}\right)=c_{2} \in\{n+1, n+2, \ldots, m\}$, we choose $x$ such that $v x$ is a valid choice for the second edge in an application of Lemma 9. This eliminates at most

$$
5 c(n)+2 P(n)+\alpha m+\beta m+4+2 \frac{k+(67+136) / n^{2}}{d} n+2 H(n)
$$


choices.

So in both cases, this choosing process eliminates at most

$$
6 c(n)+2 P(n)+2 \beta m+2 \alpha m+5+4 \frac{k+203 / n^{2}}{d} n+2 H(n)
$$

choices.

- The vertex $y$ is chosen with same strategy as $x$. Similarly, this eliminates at most

$$
6 c(n)+2 P(n)+2 \beta m+2 \alpha m+5+4 \frac{k+203 / n^{2}}{d} n+2 H(n)
$$

choices.

- $c_{1} \notin L(u y) \cup L(v x)$. This excludes at most $2 \beta m$ choices.

Thus we have at least

$$
n-\left(15 c(n)+5 P(n)+6 \beta m+4 \alpha m+10+8 \frac{k+203 / n^{2}}{d} n+5 H(n)\right)
$$

choices for an edge $x y$. Since this expression is greater than zero by assumption, we can indeed make the choice.

Now, since $M>0$, we can apply Lemma 8 or Lemma 9 to obtain a coloring where $u y$ is colored $h^{\prime \prime}\left(e_{1}\right)$. Similarly, we can apply Lemma 8 or Lemma 9 to thereafter obtain a coloring where $v x$ is colored $h^{\prime \prime}(v x)$. Next, by swapping on the 2-colored 4-cycle uvxyu, we get the proper coloring $h^{T}$ such that $h^{T}(u v)=h^{\prime \prime}\left(e_{1}\right)=c_{2}$. Since the swaps from the applications of Lemma 8 and Lemma 9 do not result in any "new" requested edges in $G_{1}$ or $G_{2}$, the swaps used in this case do not no yield any new requested edges in $G_{1}$ or $G_{2}$; similarly for all conflict edges of $K_{2 n}$.

Here, the subgraph $T$ contains the edges $u v$ and $x y$ and all additional edges used when applying the previous lemmas above; in total there are at most $2+67 \times 2=136$ edges in $T$.

Note further that besides $u v$ the only edges of $K_{2 n}$ that might be prescribed and are used in swaps for constructing $h^{T}$ are $e_{1}$ and $e_{2}$; this property shall be used when applying Lemma 11.

Case 3. $u v \in E\left(G_{1}\right)$ (or $\left.u v \in E\left(G_{2}\right)\right)$ and $c_{2} \in\{1,2, \ldots, n\}$ :

In this case, we shall construct a subgraph $T$ with at most 139 edges. Without loss of generality, we shall assume that $u v \in E\left(G_{1}\right)$. Moreover, since all requested edges with a color in $\{1,2, \ldots, n\}$ under $h^{\prime \prime}$ are in $K_{n, n}, e_{1}, e_{2} \in E\left(K_{n, n}\right)$.

If $c_{1} \in\{n+1, n+2, \ldots, m\}$, then we choose an edge $x y \in E\left(G_{2}\right)$ such that $h^{\prime \prime}(x y)=c_{1}$ and $x y$ is not prescribed or disturbed. If $c_{1} \in\{1,2, \ldots, n\}$, then we choose an edge $x y \in E\left(G_{2}\right)$ to be the first edge in an application of Lemma 10; this choice implies that $x y$ is not prescribed and not disturbed. So in both case we can have at least

$$
\left\lfloor\frac{n}{2}\right\rfloor-7 c(n)-3 P(n)-d n-2 H(n)
$$


choices for $x y$.

In addition to this, $x y$ also needs to satisfy the following:

- The edges $u x$ and $v y$ are valid choices for the second edge in an application of Lemma 7. This eliminates at most

$$
2\left(4 c(n)+P(n)+2 \beta m+2 \alpha m+5+4 \frac{k+(34+139) / n^{2}}{d} n+H(n)\right)
$$

choices.

- $c_{2} \notin L(x y) \cup \varphi^{\prime}(x) \cup \varphi^{\prime}(y) \backslash\left\{\varphi^{\prime}(x y)\right\}$. This condition will imply that after performing all swaps in this case, $x y$ is not a "new" requested edge of $G_{2}$. Since there are at most $c(n)$ edges $e$ in $K_{2 n}$ such that $h^{\prime}(e)=c_{1}$ and $c_{2} \in L(e)$, and we have already excluded the choices for $x y$ which are disturbed, this condition eliminates at most $c(n)+2(2 c(n)+P(n))$ choices.

- $c_{1} \notin L(u x) \cup L(v y)$. This excludes at most $2 \beta m$ choices.

So in total, we have at least

$$
\left\lfloor\frac{n}{2}\right\rfloor-\left(20 c(n)+d n+7 P(n)+6 \beta m+4 \alpha m+10+8 \frac{k+173 / n^{2}}{d} n+4 H(n)\right)
$$

choices for $x y$. Since this expression is greater than zero by assumption, we can indeed make the choice.

Since $M>0$, firstly if $c_{1} \in\{1,2, \ldots, n\}$, we can apply Lemma 10 to obtain a coloring where $x y$ is colored $c_{1}$. Secondly, we can apply Lemma 7 twice to obtain a coloring where $u x$ is colored $h^{\prime \prime}\left(e_{1}\right)$ and $v y$ is colored $h^{\prime \prime}\left(e_{2}\right)$. Finally, by swapping on the 2-colored 4-cycle uvyxu, we get the proper coloring $h^{T}$ such that $h^{T}(u v)=h^{\prime \prime}\left(e_{1}\right)=c_{2}$. Note that this implies that $u v$ is not a requested edge under $h^{T}$. More generally, since the swaps from the applications of Lemma 7 and Lemma 10 do not result in any new requested edges in $G_{1}$ or $G_{2}$, the swaps used in this case do not yield any new requested edges in $G_{1}$ or $G_{2}$; similarly for conflict edges in $K_{2 n}$.

Here, the subgraph $T$ contains the edges $u v$ and $x y$ and all the additional edges needed to apply the lemmas above (if we need to apply Lemma 10, then $x y$ is included in the edges used when applying this lemma); in total, $T$ uses at most $1+70+34 \times 2=139$ edges.

Case 4. $u v \in E\left(G_{1}\right)$ (or $\left.u v \in E\left(G_{2}\right)\right)$ and $c_{2} \in\{n+1, n+2, \ldots, m\}$ :

In this case we proceed similarly to Case 3, so we just sketch the arguments: using the same setup as in Case 3, a slight difference between two cases is that in Case 4, we can use Lemma 8 or Lemma 9 to obtain a coloring where $u x$ is colored $h^{\prime \prime}\left(e_{1}\right)$ and $v y$ is colored $h^{\prime \prime}\left(e_{2}\right)$. Similar calculations as above yields that we have at least

$$
\left\lfloor\frac{n}{2}\right\rfloor-\left(24 c(n)+d n+9 P(n)+6 \beta m+4 \alpha m+10+8 \frac{k+(67+205) / n^{2}}{d} n+6 H(n)\right)
$$


choices for $x y$; and this expression is greater than zero by assumption, so we can indeed make the choice and perform the necessary swaps to get the coloring $h^{T}$ satisfying $h^{T}(u v)=h^{\prime \prime}\left(e_{1}\right)=c_{2}$. Here, $T$ contains at most $1+70+67 \times 2=205$ edges.

Finally, let us note that in the first two cases, $T$ contains exactly two edges with color $c_{1}$ under $h^{\prime \prime}$. In the last two cases, $T$ contains exactly two edges with color $c_{1}$ under $h^{\prime \prime}$ if we do not have to apply Lemma 10; otherwise $T$ contains exactly three edges with color $c_{1}$ under $h^{\prime \prime}$. Any application of Lemma 7, 8 or 9 above uses at most two edges with color $c_{2}$ under $h^{\prime \prime}$, so the subgraph $T$ contains at most four edges with color $c_{2}$ under $h^{\prime \prime}$. Except $c_{1}$ and $c_{2}$, the subgraph $T$ only contains edges with colors that are not $d$-overloaded.

We will take care of every prescribed edge $e$ of $K_{2 n}$ such that $h^{\prime}(e) \neq \varphi^{\prime}(e)$ by successively applying Lemma 11; using this lemma we can construct the proper $m$-edge colorings $h_{0}=h^{\prime}, h_{1}, h_{2}, \ldots, h_{q}$, where $h_{i}$ is constructed from $h_{i-1}$ by an application of Lemma 11 and $h_{q}$ is an extension of $\varphi^{\prime}$. Since the number of prescribed edge at each vertex of $K_{2 n}$ is at most $\alpha m+c(n)$, the total number of prescribed edges in $K_{2 n}$ is at most $2 n(\alpha m+c(n))$; thus $q \leqslant 2 n(\alpha m+c(n))$.

When we apply Lemma 11, we first consider all prescribed edges $e$ in $K_{n, n}$ that satisfies $\varphi^{\prime}(e) \in\{1, \ldots, n\}$ (Case 1 in the proof of the lemma). This is important, since otherwise we might recolor such edges by colors from $\{n+1, \ldots, m\}$, and are thereafter unable to apply Lemma 11.

Thereafter we apply Lemma 11 to all prescribed edges $e$ of $K_{n, n}$ that satisfies $\varphi^{\prime}(e) \in$ $\{n+1, \ldots, m\}$ (Case 2 in the proof of Lemma 11). Note that after performing all the swaps as described in the preceding paragraph, we have not recolored any edge of $G_{1}$ or $G_{2}$. Thus, if one of the requested edges $e_{1}$ and $e_{2}$ in Case 2 of the proof of Lemma 11 is in $K_{n, n}$, then it has been used in a previous application of Lemma 11 to a prescribed edge $e^{\prime}$ of $K_{n, n}$ that satisfies $\varphi^{\prime}\left(e^{\prime}\right) \in\{n+1, \ldots, m\}$ (i.e. a "Case 2 application" of the lemma). Moreover, since the only prescribed edges that are used in an application of Lemma 11 is $u v$ and (possibly) the requested edges $e_{1}$ and $e_{2}$, it follows that every prescribed edge $e$ in $K_{n, n}$ that satisfies $\varphi^{\prime}(e) \in\{n+1, \ldots, m\}$ is not recolored by a color from $\{n+1, \ldots, m\} \backslash\left\{\varphi^{\prime}(e)\right\}$ in a "Case 2 application" of Lemma 11. Thus, we may assume that $h^{\prime \prime}(e) \in\{1, \ldots, n\}$ for any intermediate coloring $h^{\prime \prime}$ and any precolored edge $e$ in $K_{n, n}$. Hence, we can perform all the swaps as described in Case 2 in the proof of Lemma 11. Thereafter, we consider all prescribed edges of $G_{1}$ and $G_{2}$ (Cases 3 and 4 in the proof of Lemma 11).

In an application of Lemma 11 to obtain $h_{i}$ from $h_{i-1}$, we use swaps involving at most three prescribed edges: the edge $u v$, and the two adjacent requested edges $e_{1}$ and $e_{2}$. Since there are at most $2 c(n)$ prescribed edges in $K_{2 n}$ with any given color $c$ in $h^{\prime}$, there are at most $\alpha m+f(n)$ prescribed edges with color $c$ under $\varphi^{\prime}$, and $h_{i}\left(e_{1}\right)$ and $h_{i}\left(e_{2}\right)$ are not $d$-overloaded colors in the coloring $h_{i-1}$, it follows that for each $i=1, \ldots, q$, there are at most $2 c(n)+d n+\alpha m+f(n)$ edges with color $c$ under $h_{i}$ that are prescribed.

Furthermore, each application of Lemma 11 to a prescribed edge $u v$ with $h^{\prime}(u v)=c$ constructs a subgraph $T$ with at most three edges with color $c$ under $h^{\prime}$; thus a color $c$ is used at most $3(2 c(n)+d n+\alpha m+f(n))$ times in a subgraph $T$ where a prescribed edge 
has color $c$ in $h^{\prime}$. Moreover, there are at most $\alpha m+f(n)$ prescribed edges with color $c$ under $\varphi^{\prime}$, and a subgraph $T$ constructed by an application of Lemma 11 uses at most four edges with color $c$. Except for these edges, any other edges contained in a subgraph $T$ created by an application of Lemma 11 are colored by colors that are not $d$-overloaded. Hence, at most

$$
4(\alpha m+f(n))+3(2 c(n)+d n+\alpha m+f(n))+d n=7 \alpha m+7 f(n)+6 c(n)+4 d n
$$

distinct edges with color $c$ under $h^{\prime}$ are used in swaps for constructing $h_{q}$ from $h^{\prime}$.

Let $H(n)=7 \alpha m+7 f(n)+6 c(n)+4 d n, P(n)=d n+\alpha m+f(n)$; from the preceding paragraph we deduce that as long as $k n^{2} \geqslant 205 \times 2 n(\alpha m+c(n))=410 n(\alpha m+c(n))$ and

$$
\begin{gathered}
\left\lfloor\frac{n}{2}\right\rfloor-\left(2 \epsilon n+24 c(n)+6 d n+9 P(n)+6 \beta m+4 \alpha m+10+8 \frac{k+272 / n^{2}}{d} n+6 H(n)\right)>0 \\
c^{\prime}(n)=c(n) / 2 ; n-1>2 c(n)>4 ;\left(\frac{4 \beta}{\epsilon-4 \beta}\right)^{\epsilon-4 \beta}\left(\frac{1}{1-2 \epsilon+8 \beta}\right)^{1 / 2-\epsilon+4 \beta}<1 \\
\alpha, \beta<\frac{c(n)}{2(n-c(n))}\left(\frac{n-c(n)}{n}\right)^{\frac{n}{c(n)}} ; \beta<\frac{c^{\prime}(n)}{2\left(n-c^{\prime}(n)\right)}\left(\frac{n-c^{\prime}(n)}{n}\right)^{\frac{n}{c^{\prime}(n)}} \\
m-\beta m-2 \alpha m-2 c(n)-\frac{2 n c(n)}{f(n)} \geqslant 1
\end{gathered}
$$

for some constants $\alpha, \beta, \epsilon, k, d$ and functions $c(n), f(n)$ of $n$, we can apply Lemma 4 to obtain $h^{\prime}$, Lemma 5 to obtain $\varphi^{\prime}$ and finally Lemma 11 to obtain the coloring $h_{q}$ which is a completion of $\varphi^{\prime}$ that avoids $L$. This completes the proof of Theorem 2 .

\section{References}

[1] L.D. Andersen, A.J.W. Hilton, Thank Evans!, Proceedings of the London Mathematical Society, 47 (1983), 507-522.

[2] L.D. Andersen, A.J.W. Hilton, Symmetric latin square and complete graph analogues of the evans conjecture, Journal of Combinatorial Designs, 2 (1994), 197-252.

[3] L.J Andrén, On latin squares and avoidable arrays, Ph.D. thesis, Umeå University, 2010 .

[4] L.J. Andrén, C.J. Casselgren, L.-D. Öhman, Avoiding arrays of odd order by Latin squares, Combinatorics, Probability and Computing, 22 (2013), 184-212.

[5] L.J. Andrén, C.J Casselgren, K. Markström, Restricted completion of sparse Latin squares, Combinatorics, Probability and Computing, 28 (2019), 675-695.

[6] B. Barber, D. Kühn, A. Lo, D. Osthus, A. Taylor, Clique decompositions of multipartite graphs and completion of Latin squares, Journal of Combinatorial Theory, Series A, 151 (2017), 146-201.

[7] P. Bartlett, Completing $\varepsilon$-dense partial Latin squares, Journal of Combinatorial Designs, 21 (2013), 447-463. 
[8] C.J. Casselgren, K. Markström, L.A. Pham, Edge precoloring extension of hypercubes, Journal of Graph Theory, 95 (2020), 410-444.

[9] C.J. Casselgren, K. Markström, L.A. Pham, Restricted extension of sparse partial edge colorings of hypercubes, Discrete Mathematics, (2020), 343

[10] A.G. Chetwynd R. Häggkvist, Some upper bounds on the total and list chromatic numbers of multigraphs, Journal of Graph Theory, 16 (1992), 503-516.

[11] D.E. Daykin, R. Häggkvist, Completion of sparse partial Latin squares, Graph theory and combinatorics, 127-132, (Cambridge, 1983), Academic Press, London.

[12] T. Evans, Embedding incomplete latin squares, American Mathematical Monthly, 67 (1960), 958-961.

[13] J. Fiala, NP-completeness of the edge precoloring extension problem on bipartite graphs, Journal of Graph Theory, 43 (2003), 156-160.

[14] F. Galvin, The list chromatic index of bipartite multigraphs Journal of Combinatorial Theory Series B, 63 (1995), 153-158.

[15] R. Häggkvist, A solution of the Evans conjecture for Latin squares of large size, Combinatorics (Proc. Fifth Hungarian Colloq., Keszthely, 1976), Vol. I, Colloq. Math. Soc. János Bolyai, 18, 495-513.

[16] R. Häggkvist, A note on Latin squares with restricted support, Discrete Mathematics, 75 (1989), 253-254.

[17] L.A. Pham, On Restricted Colorings of $(d, s)$-Edge Colorable Graphs, Graphs and Combinatorics, 36 (2020), 853-864.

[18] B. Smetaniuk, A new construction for Latin squares I. Proof of the Evans conjecture, Ars Combinatoria, 11 (1981), 155-172. 